'Oficina de Educación Médica. Carrera de Medicina. Facultad de Medicina. Universidad Andrés Bello, sede Viña del Mar. Chile. apsicóloga. ${ }^{\mathrm{b}}$ Cirujana Dentista. 'PhD.

Los autores declaran no tener conflictos de interés. Trabajo no recibió financiamiento.

Recibido el 14 de agosto de 2018, aceptado el 4 de diciembre de 2018.

Correspondencia a: Jessica Goset Quillota $9806^{\circ}$ piso, Viña del Mar, Chile. jessicagoset@gmail.com

\section{Instrumento para el desarrollo del razonamiento clínico}

\author{
VERÓNICA SILVA ${ }^{1}$, PETER MCCOLL ${ }^{1}$, CAROLINA PÉREZ $^{1, \mathrm{a}}$, \\ MARIANA SEARLE ${ }^{1, a}$, JESSICA GOSET $^{1, b, c}$
}

\section{Development of an instrument to assess clinical reasoning}

Background: Teaching clinical reasoning is a challenge in medical education. Aim: To design a clinical reasoning assessment instrument. Material and Methods: Structured interviews were carried out to six physicians with at least five years experience. The Grounded Theory method was used to determine the relevant categories of the clinical reasoning process and the modified Delphi expert judgment method to validate the categories, the definition of observable behaviors and the format of the instrument. Results: The relevant reasoning categories were the reason for consultation, medical history, physical examination, additional tests, diagnosis, therapeutic options and reasoning reassessment capacity. Expert judgment assessed at a level of "strongly agree" and "agree" the sufficiency, clarity and pertinence of all categories, related observable behaviors and instrument format. The internal Kappa consistency yielded an index of 0.92. Conclusions: The resulting instrument was constructed with the following axes derived from the main categories and subcategories: reason for consultation, history, physical examination, additional tests, diagnosis, therapeutic options and reassessment capacity.

(Rev Med Chile 2018; 146: 1466-1470)

Key words: Clinical Decision-Making; Education, Medical.
E 1 razonamiento clínico puede considerarse un pilar fundamental en la formación médica, ya que define la pertinencia, eficacia y relevancia de la intervención del profesional, confluyendo las competencias del conocer, el hacer y el saber ser. Es la capacidad de observación, reflexión y juicio integrativo de los problemas clínicos del paciente, que permite enfrentarlos y resolverlos adecuadamente. Constituye así la competencia transversal más importante en la formación de pregrado de un médico, por lo que las escuelas y carreras de medicina deben adquirir un compromiso técnico y ético para lograr enseñarla y evaluarla de manera objetiva y reproducible ${ }^{1,2}$.

Para su enseñanza se ha recurrido didácticamente al modelado de experto, a la guía por medio de preguntas clave y a la simulación con casos predefinidos, utilizando el modelo analítico hipotético-deductivo, el modelo no-analítico de reconocimiento de patrones o una combinación de ambos ${ }^{1-3}$. Sin embargo, la enseñanza del razonamiento clínico presenta dos desfíos importantes. En primer término, los estudiantes aún no cuentan con experiencia clínica o con un conocimiento estructurado propio, lo que complejiza el reconocimiento de los patrones de enfermedad. Por ello, el contacto precoz desde los inicios de la carrera del estudiante de medicina con la problemática de la resolución de casos clínicos, ya sean simulados o reales, se vuelve crucial, pues facilitará la adquisición de la experiencia ${ }^{4}$.

En segundo lugar, a los médicos docentes les es difícil explicitar las múltiples decisiones que se toman durante el proceso, pues estas son fruto de 
la recuperación rápida de las memorias procedimentales construidas durante años de exposición a la resolución de problemas clínicos ${ }^{5}$. Mientras más integrado está el procedimiento de razonamiento clínico, más atento debe estar el docente para evidenciar los aspectos y variables que son de discernimiento casi automático para el experto, permitiendo al estudiante la identificación y ponderación de los elementos claves ${ }^{6}$. Por ello, la instancia de aprendizaje debe contar con los tiempos, espacios y estructura didáctica que permitan al académico guiar competentemente al aprendiz en la toma de conciencia del proceso.

Ya que la enseñanza del razonamiento clínico se enfoca en el proceso mental de organización del pensamiento y no exclusivamente en el logro de un resultado, es necesario que el docente favorezca la reflexión sobre el proceso de análisis clínico, guíe el debate, la retroalimentación y evidencie el avance gradual de la tarea ${ }^{7}$. Asimismo, para que esta dinámica sea fluida y eficiente, es fundamental que se promueva un clima de confianza y motivación, donde el estudiante pueda expresarse adecuadamente $^{8}$.

Cobran especial relevancia, entonces, los procesos de evaluación formativa. Para ello, el método del pensamiento hablado (thinkaloud) ofrece el espacio para conocer el proceso de elaboración que realiza el estudiante, mediante la escucha activa de su discurso en voz alta durante la resolución de un caso clínico ${ }^{9}$. Al ser usado como una estrategia didáctica transversal en las diferentes asignaturas clínicas, este método permite la exposición y reflexión de la resolución de problemas clínicos de manera repetida y continua. Se favorece así la consolidación de las estrategias para buscar y reconocer elementos claves, al mismo tiempo que la construcción de representaciones mentales de problemas clínicos.

Sin embargo, el desafío docente se mantiene si no se tiene claridad en los indicadores que permiten identificar el desempeño del estudiante durante el proceso de razonamiento clínico. Los indicadores esclarecen los objetivos de aprendizaje, otorgan transparencia a las expectativas del docente y evidencian los resultados esperados junto con los aspectos que pueden ser mejorados. $\mathrm{Al}$ estar estos claramente definidos y ser compartidos, se puede fomentar la responsabilidad y protagonismo del estudiante en su proceso de formación.

Teniendo presente todo lo anterior, la carrera de Medicina de la Universidad Andrés Bello, sede Viña del Mar, ha decidido integrar la enseñanza y la evaluación formativa del razonamiento clínico mediante la creación de un instrumento que ayude al docente en la mediación de las actividades formativas. Se espera que este constructo favorezca la sistematización de la retroalimentación inmediata durante de resolución de casos clínicos reales y facilite la puesta en común de los procesos mentales inherentes al razonamiento clínico. Se pretende así promover que los docentes puedan centrarse en la apropiación del proceso de razonamiento clínico del estudiante y no en el resultado final del razonamiento propiamente tal. Para ello se definieron las conductas observables que reflejen los indicadores para cada una de las categorías relevantes del razonamiento clínico.

\section{Material y Método}

Esta investigación tiene un diseño cualitativo, ya que se pretende comprender el proceso de razonamiento clínico desde la visión del experto que cumple rol docente. Para generar los datos, se realizaron entrevistas semiestructuradas a un grupo de 6 médicos cirujanos que contaran con a lo menos 5 años de experiencia clínica ininterrumpida, grupo que permitió saturar la muestra. El análisis fue realizado mediante la técnica de teoría fundamentada en su nivel descriptivo ${ }^{10}$. La identificación de los códigos en vivo permitió definir categorías relevantes y subcategorías. Las categorías identificadas para la construcción del instrumento se triangularon con notas y observaciones de campo de las instancias de enseñanza-aprendizaje de razonamiento clínico, junto con el análisis del material de apoyo que se les entregaba a los estudiantes.

La segunda etapa de la construcción del instrumento corresponde a su validación por medio del método juicio de experto Delphi modificado. Se seleccionó este método, ya que respeta la pluralidad de la información y rescata la cualidad de las respuestas de los expertos, los que fueron seleccionados mediante coeficiente de competencia experta. A los jueces se les entregó la planilla de valoración con escala de Likert de 4 niveles para cada pregunta. Esta fue elaborada según la propuesta de Escobar y Cuervo ${ }^{11}$, considerando las variables suficiencia, coherencia, relevancia y claridad y el 


\section{Tabla 1 Categorías y conductas observables}

\begin{tabular}{|ll|}
\hline Categorías & Conductas observables \\
\hline Motivo de la consulta & $\begin{array}{l}\text { Declara el motivo de consulta del paciente de acuerdo a lo que él o sus parientes expresan. } \\
\text { En el contexto de edad y sexo debe plantear las primeras opciones a investigar y/o diagnósticos } \\
\text { probables }\end{array}$ \\
\hline Anamnesis & $\begin{array}{l}\text { Explica cómo efectuó la anamnesis completa. En base a los datos obtenidos el estudiante afian- } \\
\text { za o corrige las opciones generadas en el paso 1. Él debe plantear una nueva lista de opciones } \\
\text { diagnósticas. Debe explicar y ponderar los datos de la anamnesis }\end{array}$ \\
\hline Exploración física & $\begin{array}{l}\text { Explica cómo efectuó el examen físico completo, general, sistemático y dirigido a área específica } \\
\text { de acuerdo al motivo de consulta. Debe re-elaborar lista de probables diagnósticos en base a lo } \\
\text { obtenido en los pasos 1 y 2. Debe explicar y ponderar y los hallazgos del examen físico }\end{array}$ \\
\hline Exámenes & $\begin{array}{l}\text { Explica los exámenes de laboratorio e imagenológicos considerados que apunten a la confirma- } \\
\text { ción o descarte de las opciones diagnósticas planteadas en el paso anterior. Debe describir de } \\
\text { manera completa los exámenes de imágenes y de laboratorio }\end{array}$ \\
\hline Opiagnóstico & $\begin{array}{l}\text { Define el diagnóstico o decide si es necesario confirmarlo con exámenes más invasivos, inclu- } \\
\text { yendo punciones, biopsia u otros. La fundamentación del diagnóstico final se debe basar en un } \\
\text { resumen de los hechos centrales que lo sustentan }\end{array}$ \\
\hline Evolución tratamiento & $\begin{array}{l}\text { Expone la decisión sobre las opciones terapéuticas, médicas o quirúrgicas a utilizar } \\
\text { Capacidad reevaluadora el análisis del resultado de los tratamientos y el curso evolutivo del caso }\end{array}$ \\
\hline
\end{tabular}

espacio para las observaciones pertinentes. Para determinar la consistencia interna de las respuestas de los jueces se utilizó el índice Kappa.

\section{Resultados}

El análisis cualitativo determinó para el razonamiento clínico dos categorías principales asociadas a linealidad y recursividad del proceso. La primera categoría presentó subcategorías relacionadas con conocimiento del caso, antecedentes próximos y remotos, exámenes para el diagnóstico y opciones de tratamiento. La categoría de recursividad no presentó subcategorías.

Tras la triangulación de la información con las notas de campo-observación y análisis del material guía entregado a los estudiantes, se construyó el instrumento con las siguientes variables derivadas de las categorías principales y subcategorías: motivo de consulta; anamnesis; exploración física; exámenes; diagnóstico; opciones terapéuticas y capacidad reevaluadora (recursividad).

El juicio de experto valoró en un nivel de "muy de acuerdo" y "de acuerdo" la suficiencia, claridad, coherencia y relevancia de todas las variables, conductas observables relacionadas y formato del instrumento, arrojando un índice de consistencia interna Kappa de 0,92. LaTabla 1 especifica las conductas observables identificadas para cada variable.

\section{Discusión}

El razonamiento clínico es una competencia clínica intrínsecamente contextual que se desarrolla con la práctica, la reflexión sobre la experiencia y responde a la capacidad de recuperación del conocimiento y la organización del pensamiento durante el análisis hipotético causal ${ }^{6-12}$. Sin embargo, a pesar de la experticia del docente en esta competencia clínica, existe la dificultad para explicar cuál es el proceso mental que se lleva a cabo para obtener una conclusión diagnóstica y por lo tanto mediar el aprendizaje de esta habilidad ${ }^{13}$.

Un mecanismo para guiar el rol docente en el desarrollo de las habilidades de pensamiento crítico del estudiante, la reflexión, autoevaluación y retroalimentación, es el uso de instrumentos 
didácticos ${ }^{14}$. La construcción y utilización de un instrumento como guía para el estudiante, autoevaluación y evaluación formativa del proceso de razonamiento clínico favorece la retroalimentación sistematizada, elemento crucial para el aprendizaje de esta competencia clínica transversal ${ }^{15}$.

En este trabajo las categorías levantadas que dan paso a la creación de los indicadores del instrumento son coherentes con el planteamiento del razonamiento clínico cómo una competencia que se basa en habilidades lógico-intelectuales del identificar, contrastar, ponderar, interpretar, reflexionar, evaluar y predecir.

La categoría relacionada con el componente lineal concuerda con lo descrito por Montaldo y Herskovic $^{16}$, quienes utilizan el reconocimiento de patrones de enfermedades o Illness Script (IS). En él, el método clínico, o "proceso del diagnóstico", incluye los pasos ordenados que se aplica en la búsqueda del diagnóstico mediante obtención de la información necesaria (síntomas, signos y otros datos); generación de las hipótesis diagnósticas presuntivas; comprobación de dichas hipótesis y, finalmente, emisión del diagnóstico definitivo de certeza e indicación del tratamiento.

Asimismo, el componente recursivo concuerda con el planteamiento de que el razonamiento clínico requiere de discusión diagnóstica ${ }^{17}$. Así, lejos de ser una habilidad reproductiva, el razonamiento clínico involucra procesos mentales complejos, en ocasiones controversiales, pues involucra plantear y replantear diversas hipótesis, hasta lograr comprobarlas ${ }^{18}$, siendo el componente cíclico lo que permite reconocer el hallazgo inesperado necesario para las reformular hipótesis y redireccionar la activación de los compilados de conocimiento hasta lograr aceptar un patrón de enfermedad dado ${ }^{19}$. Tal como lo mencionan Montaldo y Herskovic ${ }^{16}$, el razonamiento clínico constituye el principal método de interacción clínica con el paciente, y supone conocimiento, reflexión y acción.

Esta investigación pretende ser un aporte a la enseñanza, aprendizaje y evaluación de esta competencia fundamental en la formación médica de nuestro tiempo. De esta manera se espera contribuir a la toma de conciencia del proceso de razonamiento clínico por parte del estudiante, facilitando su aprendizaje al ser utilizado como elemento guía en los procesos de reflexión, autoaprendizaje, devolución constructiva y evaluación formativa.

\section{Referencias}

1. Villarroel J, Ribeiro Q, Bernal N. Razonamiento Clínico: Su Déficit Actual y la importancia del aprendizaje de un Método durante la formación de la Competencia Clínica del Futuro Médico. Rev Cient Cienc Méd 2014; 17 (1): 29-36.

2. Croskerry P. A universal model of diagnostic reasoning. Acad Med 2009; 84: 1022-8.

3. Corona L, Fonseca M. El razonamiento diagnóstico en el método clínico. La comparación y otros procesos mentales como herramientas del juicio clínico. MediSur 2012; 10 (1): 39-46.

4. Del Angel GM, Arteaga F. Utilidad de una estrategia educativa activo-participativa en el desarrollo del razonamiento clínico en pregrado. Educ Med Super 2015; 29 (2): 264-72.

5. Cardozo M, Alzate O. Bases moleculares de la memoria y su relación con el aprendizaje. Arch Med (Manizales) 2016; 16 (2): 467-84.

6. Gormaz C, Brailovsky C. Desarrollo del razonamiento clínco en medicina. REDU: Revista de Docencia Universitaria 2012; 11 (1): 177.

7. Rivera M, Pernas M, Nogueira M. Un sistema de habilidades para la carrera de Medicina, su relación con las competencias profesionales. Una mirada actualizada. Educ Med Super 2017; 31 (1): 215-138.

8. Mora F. Neuroeducación. Madrid: Alianza Editorial 2017.

9. Norman G. Research in clinical reasoning: past history and current trends. Med Educ 2005; 39 (4): 418-27.

10. Strauss A, Corbin J. Bases de la investigación cualitativa. Técnicas y procedimientos para desarrollar la teoría fundamentada. Editorial Universidad de Antioquia 2002.

11. Escobar J, Cuervo A. Validez de contenido y juicio de expertos: una aproximación a su utilización. Avances en medición 2008; 6 (1): 27-36.

12. Rodríguez A, Valdés C, García C, Rodríguez L. Habilidades de razonamiento clínico en estudiantes de la carrera de Medicina. Humanidades Médicas 2013; 13 (2): 433-56.

13. Coderre S, Mandin H, Harasym PH, Fick GH Diagnostic reasoning strategies and diagnostic success. Med Educ 2003; 37 (8): 695-703.

14. Cano LM, de Sarmiento MO, Encizo S, Bernal G. El uso de las rúbricas en el desarrollo del razonamiento hipotético-deductivo en estudiantes de medicina en una universidad colombiana. Entramados: educación y sociedad 2017;(4): 185-200.

15. Groopman J. How doctors think. New York: Houghton Mifflin 2007. 
16. Montaldo G, Herskovic P. Aprendizaje del razonamiento clínico por reconocimiento de patrón, en seminarios de casos clínicos prototipos, por estudiantes de tercer año de medicina. Rev Med Chile 2013; 141 (7): 823-30.

17. Silva V, Ortega J, McColl P, Searle M, Pérez C, Escobar $\mathrm{V}$. Elementos de la didáctica en la cirugía en pregrado: un estudio cualitativo. Inv Ed Med 2017; 7 (25): 53-60.

18. Losada JL, Hernández E. Apreciaciones acerca de la enseñanza del método clínico. Gaceta Médica Espirituana 2009; 11 (2): 1-13.

19. Meterissian SH. A Novel Method of Assessing Clinical Reasoning in Surgical Residents. Surg Innov 2006; 13: $115-9$. 